\title{
Towards Predictive Maintenance for Flexible Manufacturing Using FIWARE
}

\author{
Go Muan Sang ${ }^{1}$, Lai $\mathrm{Xu}^{1}$, Paul de Vrieze ${ }^{1(\bowtie)}$, and Yuewei Bai ${ }^{2}$ \\ ${ }^{1}$ Faculty of Science and Technology, Bournemouth University, \\ Poole, Dorset, UK \\ \{gsang, 1xu, pdevrieze\}@bournemouth.ac.uk \\ 2 Industry Engineering of Engineering College, \\ Shanghai Polytechnic University, Shanghai, China \\ ywbai@sspu.edu.cn
}

\begin{abstract}
Industry 4.0 has shifted the manufacturing related processes from conventional processes within one organization to collaborative processes across different organizations. For example, product design processes, manufacturing processes, and maintenance processes across different factories and enterprises. This complex and competitive collaboration requires the underlying system architecture and platform to be flexible and extensible to support the demands of dynamic collaborations as well as advanced functionalities such as big data analytics. Both operation and condition of the production equipment are critical to the whole manufacturing process. Failures of any machine tools can easily have impact on the subsequent value-added processes of the collaboration. Predictive maintenance provides a detailed examination of the detection, location and diagnosis of faults in related machineries using various analyses. In this context, this paper explores how the FIWARE framework supports predictive maintenance. Specifically, it looks at applying a data driven approach to the Long Short-Term Memory Network (LSTM) model for machine condition and remaining useful life to support predictive maintenance using FIWARE framework in a modular fashion.
\end{abstract}

Keywords: Predictive maintenance $\cdot$ FIWARE $\cdot$ LSTM $\cdot$ Big data analytics $\cdot$ Industry 4.0

\section{Introduction}

Modern complex and competitive manufacturing demand flexible and modular systems to optimize production processes; maintenance and market demands; and to support collaborative partners $[1,2]$. In the context of Industry 4.0 , utilizing emerging technologies such as Internet of things, advanced data analytics and cloud computing provides new opportunities for flexible collaborations as well as effective optimization of manufacturing related processes, e.g. predictive maintenance [1].

Both operation and condition of the production equipment are critical to the whole manufacturing process $[3,5]$. Failures of the machine tools easily can have impact such as delay on the subsequent value-added processes of the organization, partners and its customers, due to the interlinked nature of production systems [4, 5]. Essentially, any 
unplanned failure or inefficient process of manufacturing equipment can result in an unplanned downtime and costs for an entire production line [3, 5]. Traditional processes dealing with maintenance are complex, and costly [6]. It is impossible for the traditional data processing approaches and tools to produce meaningful information from the huge volume of data generated by modern manufacturing processes [7].

Condition-based maintenance utilizes condition measurements to schedule appropriate maintenance activities while minimizing impact to normal machine operations $[3,5]$. In order to achieve optimal maintenance decision making, a new approach should be in place to utilize multiple data sources from different data domains. Typically, production data, machine operation and function data, and sensor data are all required for the analysis (real-time, off-line) and used to build models for predicting machine condition i.e. failure, worn, etc., or inefficient process or poor product quality reducing failure times and costs $[3,5,6]$. Real-time monitoring of machine tools and equipment together with predictive models, visualization and data analysis supported by flexible predictive maintenance platform can lead to effective maintenance.

This paper presents an approach for data driven predictive maintenance of machine equipment, based on real-time shop floor monitoring and enhances the collaboration between parties involved through flexible maintenance platform in the context of Industry 4.0 by applying FIWARE framework, enabling modularizing of related functions. The contributions of this work are a) design a predictive maintenance analytics platform based on FIWARE b) propose data-driven approach with Long ShortTerm Memory (LSTM) network for RUL estimation, which can make full use of the sensor sequence data, and c) using the design predictive maintenance platform to present the application case.

\section{Related Work}

The emerging Industry 4.0 drives the focus of modern industrial collaborative computing [1]. Industry 4.0 can be realized as the flexibility that exists in value-creating networks is increased by the application of emerging technologies such as the internet of things, Cyber Physical Systems (CPS), cloud computing, enabling machines and plants to adapt their behaviors to changing orders and operating conditions through self-optimization and reconfiguration [1]. Essentially the data exchanged and produced in such interaction among several components establishes the underlying business processes for collaboration. Collaborative business processes are required being moved across factories and enterprises to effectively manage and ease the life cycle of production and its demands [8,9]. This requires a flexible and modular platform. Furthermore, with the demand for data to flow across different collaborative domains, new important challenge like transparency and traceability arise [4].

Effective maintenance is essential to decreasing the costs associated with downtime and faulty products in highly competitive and complex manufacturing industries [3, 4]. In the context of predictive maintenance, remaining useful life (RUL) estimation and detecting the tool condition of an equipment enable to schedule effective schedule plan in advance, avoiding unexpected failure, ensuring smooth replacement maintenance, cancelling unnecessary maintenance to reduce cost and adjusting the operating 
conditions, such as speed, load, to prolong the life of the equipment $[3,5,6]$. RUL of a component or a system is defined as the length from the current time to the end of the useful life whereas tool condition can be described as degradation (worn-out) or health of machine equipment $[5,6,10]$.

The condition and health of the production machine is critical to the whole manufacturing process [3-5]. To support this, traditional maintenance approaches such as manual and fixed maintenance scheduling are typically carried out [3]. However, this approach is cumbersome, costly and introduces the possibility of human error [5, 6]. The continuous collection of large amounts of data from sources such as sensors and equipment usage can provide new opportunities to operations and maintenance process to be proactive with ongoing equipment maintenance and upkeep [3, 5]. This enables optimization of the operation and condition of the equipment as well as predict future potential issues in a system or equipment and, therefore utilize maintenance in a predictive manner $[3,5]$.

Data-driven with machine learning approaches are recognized in providing the rising effective solutions in facilitating the decision-making process, assisted by the advanced capabilities of cloud computing, big data and analytics [7]. There however exist challenges in predictive maintenance; the complexity and the capacity to manage big data with the nature of being dynamic and complex associations, and the flexibility and interoperability to integrate different systems/components [2, 5, 9]. Several conceptual frameworks for predictive maintenance have been proposed in the research community [11-14]. However, key factors such as modular design i.e. to act easily and dynamically based on needs, advanced analytics and middleware capability based on the context of Industry 4.0 standards, are still overlooked. In order to achieve a flexible predictive maintenance with an optimal maintenance decision making, a new approach should be in place to support the integration of different components, multiple data sources from different data domains as well as advanced analytics capabilities in a modular fashion.

\section{Predictive Maintenance}

\subsection{FIWARE Architecture}

FIWARE is an open source platform for building smart solutions in different application domains [15]. It offers a catalogue library of components known as Generic Enablers (GEs), along with a set of reference implementations that allow the instantiation of some functionalities such as Big Data analysis, development of context-aware applications, connection to the Internet of Things [15]. Existing architecture such as 5level approach [11] generally focuses on design architecture and lacks consideration for modularity required for flexible predictive maintenance. On the other hand, FIWARE is a modular and open sourced platform, third parties or other software as required can be integrated via a plug in/out option [15], and hence is adopted in this work. In addition to acquiring modular feature, an architecture for predictive maintenance based on the concept of big data analytics and cloud computing is considered in this work. From the analysis and architecture of big data analytics systems in our previous work [7], the 
underlying functions and processes of the big data systems such as data collection, processing, modeling and analysis, and visualization, are realized for designing big data analytics required for predictive maintenance.

The proposed architecture generally consists of four main layers. The first layer is an application layer concerning applications, interfaces, dashboards, etc. The second layer is a process layer involves various processes. The third layer is a middleware and data layer concerning broker, adapters, data storage. The fourth layer contains a resource layer referring to factory resources e.g. production machines/equipment. Each layer has a general focus but is flexible enough to make up of different requirements i.e. processes, systems, tools, to meet different requirements. The architecture is controlled by identity access and management.

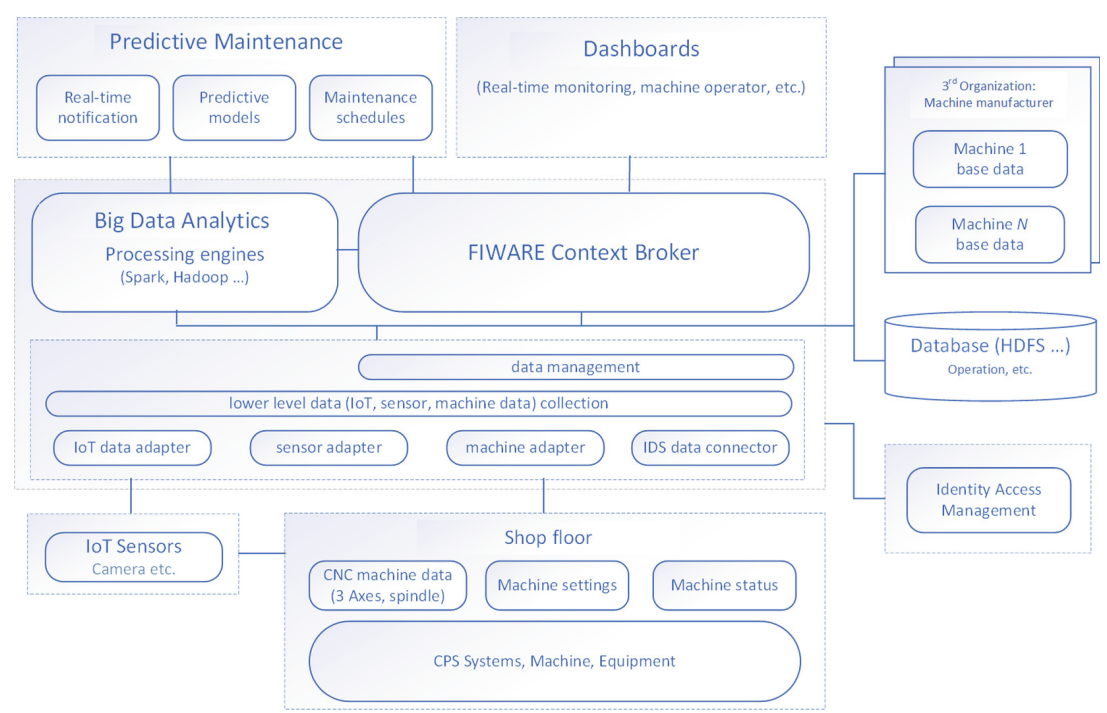

Fig. 1. Proposed FIWARE architecture platform.

At the resource layer i.e. the shop floor in Fig. 1, the framework gathers data from the shop floor, deployed sensors or devices deployed in the shop floor, which contains for example, operational machine data such as $\mathrm{CNC}$ operation data and spindle, CNC machine data, CNC assistant data, predefined CNC setting, machine statues, etc.

At the middleware and data layer, the FIWARE context broker acts as the core middleware in a publish and subscribe manner, accommodating the interaction of different processes, APIs, systems for the whole platform. Lower level data is collected using different data adapters, injecting into the Big data analytics processing engines for the process layer. Collected data is kept in storage like HDFS as required. In addition, machine measurement and basic machine data from the manufacturer is stored and managed in the industrial data space.

The process layer is implemented by the FIWARE framework, integrating different modules and functionalities required for predictive maintenance. The capability of big 
data analytics is essential for operating predictive maintenance in modern manufacturing described in Sect. 2. Thus, the core function of the proposed predictive maintenance platform is big data analytics, enabling big data analytics including batch and streaming data analytics processing incorporating with different data from sensor, Hadoop Distributed File System (HDFS), the platform database and other related manufacturing systems. The analytics can be available via the context broker's APIs for the functionalities of the application layer as a publish/subscribe manner.

For the application layer, the FIWARE context broker will collect data from different resources via various adapters or sensor enabled devices. This is used to fill the top-level Dashboards for monitoring purposes as well as inform predictive maintenance modules for maintenance scheduling purposes.

\subsection{Predictive Maintenance Methods}

As Industry 4.0 associates with IoT, big data and cloud computing, data driven approach is the cost-effective option compared with the model-based and experiencebased approaches which are highly complex, difficult to build and maintain [6]. Hence, data driven approach is adopted in this work. A general data driven predictive maintenance approach includes data acquisition, data preprocessing, development of detection or prediction model and deployment and integration of the developed model [16]. In the case of Industry 4.0, multiple different machines/equipment involve in the production chain, hence further considerations such as multiple resources, machines, data fusion and processing, etc., are recognized.

In the case of RUL and tool wear detection aspects such as failure, degradation, various data such as manufacturing machine/equipment operational data collected via sensor are used to build models, capturing time sequence information [5, 10]. Methods such as sliding window, Hidden Markov Model (HMM) and Recurrent Neural Network (RNN) are widely used [10]. However, these approaches face challenges; computational complexity and storage with HMM, memory problems with RNN [17, 18]. Long Short-Term Memory Network (LSTM), a type of RNN, overcomes the memory problems by controlling information flow using gates (input, forget and output) [19]. Due to the nature of sequential sensor data, LSTM is suitable for data driven predictive models for machine condition and RUL. The approach for learning LSTM model in this work is presented in Fig. 2. The step can be iterative i.e. evaluate model to train model, etc., and adjusted to different needs i.e. parameter or network settings, autoencoder, etc., as required to the learning purpose.

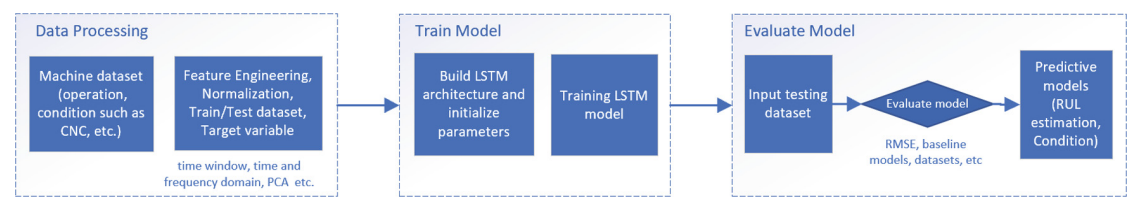

Fig. 2. Procedure of LSTM model 
To support the aspect of predictive maintenance within the Big data analytics of the process layer, the predictive models in Fig. 1, combining data derived from the machine tool operator, manufacturer and sensors are used for developing the predictive models following the steps in Fig. 2, and are predicting the machine condition (e.g. worn) and remaining useful life (RUL) $[5,6,10]$.

As in the case of real-time monitoring and notification in Fig. 1, the underlying machines, devices and factories are considered as the maintenance items. During operation, real-time state data collected from the underlying machine is processed by comparing the key state of each maintenance item including the threshold [5]. The state and threshold of the equipment item is stored in the maintenance repository in a database. Based on the notification of machine condition and RUL, maintenance schedule can be planned, and appropriate tasks can then be decided and performed for optimal operation.

\section{FIWARE Predictive Maintenance for Application Case}

A flexible manufacturing factory consists of a processing system, a logistics system, an information system, and an auxiliary system, collaborating multiple partners and customers. A concrete scene of the flexible factory is shown in Fig. 3. The processing system in the scene consists of 4 sets of equipment, which consists of an automatic stereoscopic warehouse, numbers of AGV trolleys, three robots, numbers of frames of carrier plates. Coordinate measuring machine (CMM) is responsible for the measurement. A cleaning machine and a drying machine are responsible for cleaning and drying the workpiece. Production data are typically generated by different machines equipment operation, condition, setting, etc., including $\mathrm{CNC}$ machine tool.

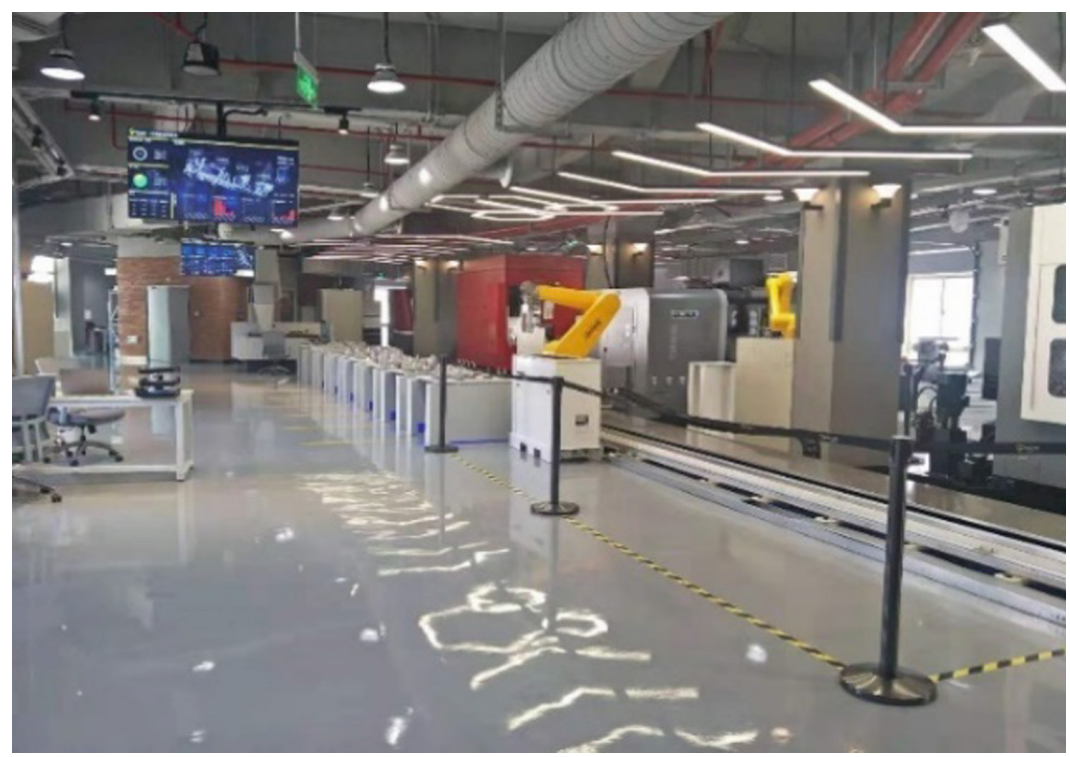

Fig. 3. Flexible manufacturing factory [5] 
In the context of flexible manufacturing in this case, data are collected from different machines and tools via sensor as well as other information systems like ERP, MES, etc. Also, the manufacturing industries work beyond their boundary i.e. collaborative partners, suppliers, etc., and the nature of dynamic data can be extremely frequent and highly voluminous in the context of modern factory $[1,2,5]$. Thus, it is essential to use a consistent but extensible model to allow for a flexible predictive maintenance in manufacturing. FIWARE offers a data model based on an entityattribute model, the entities are used to represent and model real world objects including virtual entities, the attributes that describe different aspects of the entity as well as supporting open standards and extensibility [15]. In this aspect, FIWARE model meets the requirements of flexible predictive maintenance platform, thus it is adopted for the application case as presented in Fig. 4.

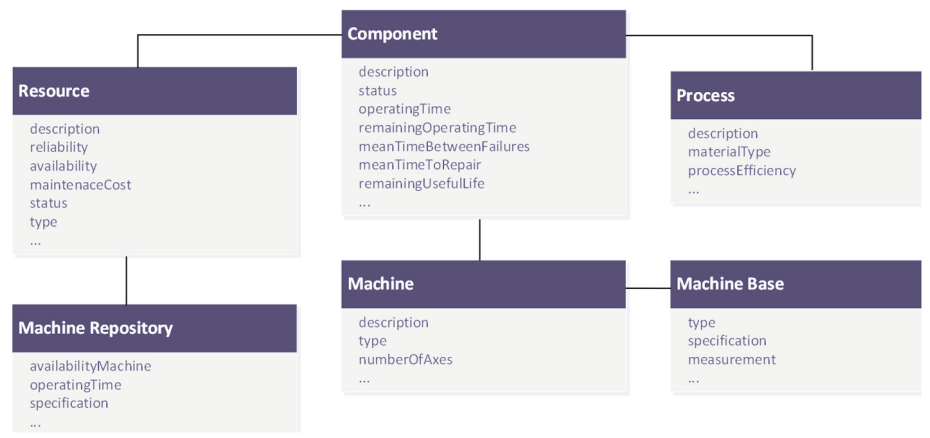

Fig. 4. Sample machine data model

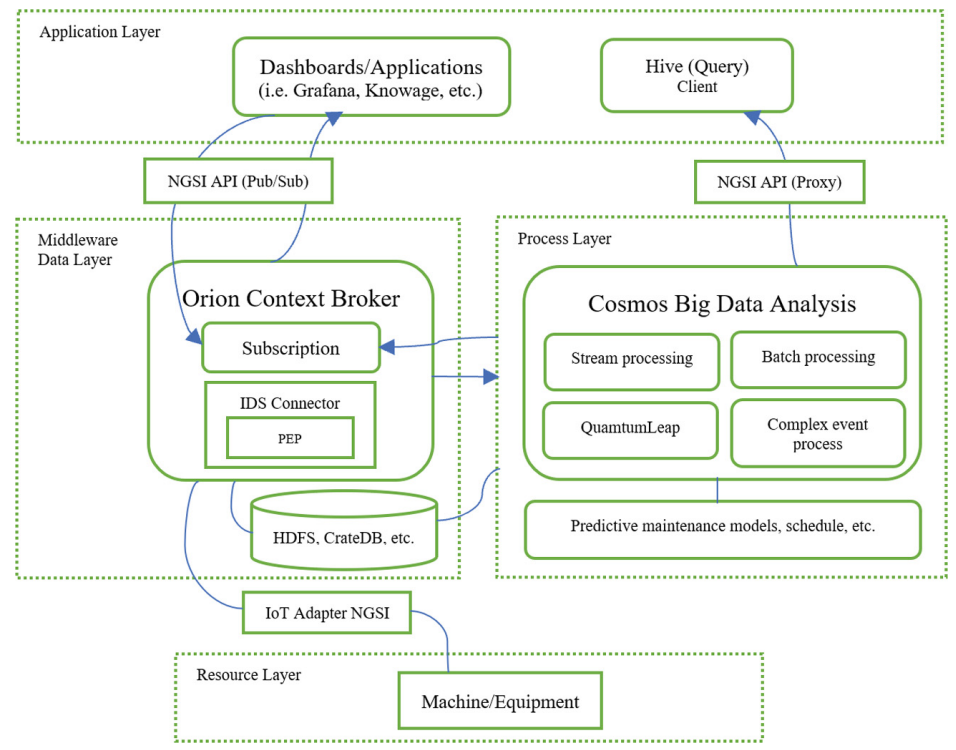

Fig. 5. Propose FIWARE predictive maintenance Case 
The proposed architecture in Fig. 1 is instantiated for the application case in Fig. 3 using key components enabling Big Data Analytics within FIWARE framework in Fig. 5 with the LSTM predictive models in Sect. 3.2 and data model in Fig. 4.

At the resource layer, different machines/devices are used for production, connected via associated adapters enabling the interaction with the context broker and related data processes and storage using the proposed data model. For seamlessly connecting, managing and gathering data of IoT devices in this case, FIWARE generic enablers such as IoT Agent for JSON, and OPC-UA, as well as Ultralight 2.0 protocol and NGSI for complex real-time processing are configured as required.

For the middleware and data layer, Orion Context Broker as the middleware represents a Publish/Subscribe Context Broker Generic Enabler hosted within the predictive maintenance platform. It provides services including the registration of provider systems, updates and notifications based on changes in context information, and query of context information [15]. The Orion context broker utilizes the NGSI REST API and PEP Proxy, which provides a connector that enables the context data coming from the Context Broker to be pushed into HDFS storage as the data layer enforcing security policy. Following the big data systems architecture and FIWARE open standards [7, 15], collected data are kept into the platform Data Lake enabling a central source for processing and analytics.

In the context of process layer, the Cosmos Big Data Analysis Generic Enabler enables Big Data analysis of both batch and stream data. It includes a Hadoop engine, an authentications generator based on NGSI API Oauth2, and a connector to the Context Broker [21]. Data is injected into the Big Data GE's HDFS by either accessing the integrated Hadoop command line interface via a shell, or by using Telefonica's SSH File Transfer Protocol (SFTP) server for direct data injection into HDFS [21, 22]. The results from MapReduce will be accessed via HDFS (input and output folders created) [21, 22]. The processed data is then made available to the context broker. For real-time analytics, QuantumLeap with the ability to configure rules on the complex event process allows instant time-series data pushed by machine equipment, which then can be available, for example via Gafana at the application layer.

For the application layer, different dashboards, applications and other user interfaces can be integrated upon requirements. In this case, dashboards such as Grafana offers to view or notify real-time data generated by machine equipment. In addition, Hive (SQL) query is supported for ad hoc query within the big data analytics module $[15,21]$.

Regarding predictive model, a sample dataset is initially trained, following the procedure in Fig. 2. At this stage, it is the initial training from a sample dataset 12000 with 30 time step to process a batch of 28 variables. Initial batch size of 750 was used. The number of training epochs was set at 71 epochs but was increased for datasets where the algorithm required a longer time to converge. A dropout rate of $20 \%$ were used after the LSTM. The model is trained using Keras library with TensorFlow backend [20]. All models were trained via the Adam optimizer. The sample data and initial sample result are presented in Fig. 6 and Fig. 7 respectively. Full analysis and model learning will be carried out as future work in order to achieve optimized models for production deployment. 
In addition to utilizing LSTM predictive models, actual operating time can be calculated, and the remaining operating time until maintenance is subsequently determined using the proposed data model in Fig. 4 [5, 23]. Furthermore, the remaining operating life of the machine equipment can be calculated by the extended Taylor's equation, obtaining the values of the parameters including the speed, the feed rate, and the properties of the machine tool material and the workpiece as well as KPIs [23].

The information about the machine equipment stored in the machine repository proposed in the data model is available via the dashboard, which is also accessible to the digital twins for available machine equipment. This includes, the design of each machine and its configuration capability such as the workpiece, etc., and the information related to the available machines and their parameters. This enables the maintenance operators who create the process plan, obtaining the information about the availability of the resources for creating viable process plans via the dashboard.

To support transparent collaboration in the proposed platform, machine base data is accessed via the FIWARE's IDS connector, facilitating the transparency of policy, data flow, usage and access across the interactions between each component with the context broker on the platform. To gain greater scalability of the proposed architecture, FIWARE offered container virtualization using container images is adopted for deployment [24].

\begin{tabular}{|l|l|}
\hline Feature & Data Type \\
\hline Machine process & int \\
\hline Machine sequence number & int \\
\hline Machine & int \\
\hline Machine condition & int \\
\hline Feed rate & float \\
\hline Camp pressure & float \\
\hline Axis $(\mathrm{X}, \mathrm{Y}, \mathrm{Z})$ & float \\
\hline
\end{tabular}

(a)

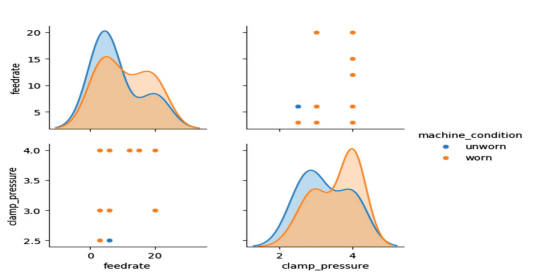

(b)

Fig. 6. (a) Sample machine dataset (b) Sample machine operational data
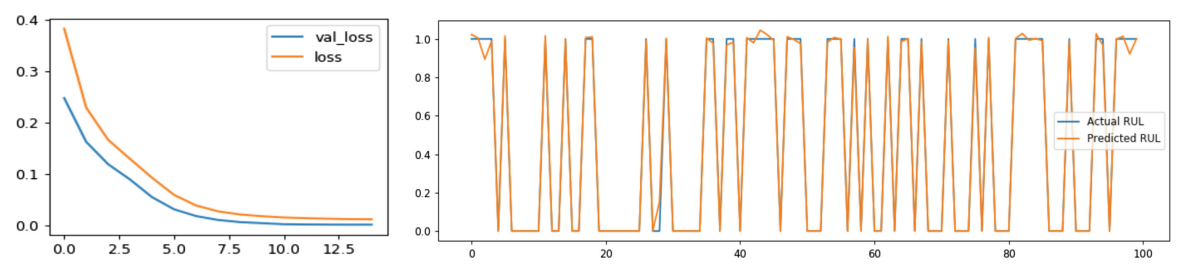

Fig. 7. Initial result from sample dataset

\section{Discussion}

Modern manufacturing such as the application case in Fig. 3 is complex and involves modern plant-run machines deployed smart sensors (sensor networks), network of collaborative partners and processes, and robots running on the shop floor. Flexibility is 
critical for the whole production chain, in the sense that the existing systems e.g. machines can easily be integrated with predictive maintenance, and other different systems or processes without significant effort. In other words, interoperability i.e. the ease integration of the existing machines, robots, systems, etc. and predictive maintenance platform is highly important. Existing approach [11] offers 5-level architecture for Industry 4.0 manufacturing systems, however it lacks the consideration for modularity, collaboration, middleware component or big data analytics implementation.

The proposed solution however aims to provide flexible and modular architecture using FIWARE framework in Fig. 5. The flexibility is achieved by facilitating the ease integration of different components required for predictive maintenance. The proposed 4-layer approach, like 5-level approach [11] supports better understanding of different components/processes at different levels as well as the overall architecture. Our adoption of FIWARE framework also provides the modularity which facilitates the ease integration i.e. interoperability of different components as pluggable components. In this sense, the case's current different machines, robots, systems and other information systems like MES, ERP, Logistics, CRM, etc., can be easily integrated with the designed predictive maintenance solution, enabling effective maintenance analytics with minimal effort.

Besides, middleware technology which is not considered in existing 5-level architecture [11], is the basis for any IoT system to manage effective communications/ interactions i.e. machine to machine, machine to system, etc. within a context. The adopted FIWARE context broker along with Big Data Analysis module facilitates the support for the interaction and integration of existing as well as future different IoT devices within the production plant as well as other collaborative context for information analytics.

In the context of collaboration in the described case, various collaborative processes exist in the form of network of machines and systems as well as network of collaborations i.e. machine manufacturers, suppliers, insurers, customers, etc. In other words, data interaction occurs at different levels i.e. components, machines, systems. Also, collaborative data are moved/accessed; machine base data from manufacturer, product design data from partner as well as machine status/diagnosis required to the insurer for purposes e.g. claim analysis. The application of IDS connector along with the proposed data model facilitates greater interoperability as well as greater transparency of data access due to the policy and usage controls of the IDS connector in a collaborative context. Ultimately, it will facilitate virtual factory production mode which requires a higher level of integration of data from customers, suppliers and partners across enterprises, enabling the optimization of the information flow and delivery process for smart plant information systems.

In the case of maintenance in the presented case, effective maintenance is essential to decreasing the costs associated with downtime and faulty products. The proposed solution currently focuses on predictive maintenance in the manner that maintenance activity can be carried out before a potential failure or a degradation of a machine/equipment is detected. On the other hand, without the process of optimal maintenance schedule plan for the existing complex machines/equipment within the production chain, it is not easy to manage effective maintenance. In this regard, optimal 
maintenance schedule plan considering complex systems, cost, etc., in the context of Industry 4.0 , will be hugely significant for future work.

\section{Conclusion}

In this paper, we proposed flexible and modular Predictive Maintenance using FIWARE to overcome some challenges of predictive maintenance in modern collaborative manufacturing. Predictive maintenance in industry collaborations in the complex and dynamic manufacturing environment requires a concrete, extensible architecture and platform. Unlike the 5-level approach, the proposed predictive maintenance platform offers flexible and modular system with advanced capabilities to handle the requirements of Industry 4.0 and advanced analytics e.g. LSTM models (machine condition, RUL). The effectiveness of the proposed solution is demonstrated within a flexible manufacturing case. Using predictive maintenance analytics, the maintenance task can be performed in an efficient manner to avoid unnecessary downtime, to keep low cost and to provide better management of the condition and process of expensive manufacturing equipment and optimization of the whole production chain. Regarding future work, we plan to do the implementation and evaluation of the design platform including predictive models, with the described application case and further use cases across industries as well as the development of optimal predictive maintenance schedule plan considering complex systems of Industry 4.0.

Acknowledgements. This research is partially funded by the State Key Research and Development Program of China (2017YFE0118700) and it is part of the FIRST project which has received funding from the European Union's Horizon 2020 research and innovation programme under the Marie Skłodowska-Curie grant agreement No 734599.

\section{References}

1. Thoben, K.D., Wiesner, S., Wuest, T.: "Industrie 4.0" and smart manufacturing-a review of research issues and application examples. Int. J. Autom. Technol. 11(1), 4-16 (2017)

2. Koren, Y., Gu, X., Guo, W.: Reconfigurable manufacturing systems: principles, design, and future trends. Front. Mech. Eng. 13(2), 121-136 (2018). https://doi.org/10.1007/s11465018-0483-0

3. Mobley, R.K.: An Introduction to Predictive Maintenance. Butterworth-Heinemann, Oxford (2002)

4. Wang, L.: Machine availability monitoring and machining process planning towards cloud manufacturing. CIRP J. Manuf. Sci. Technol. 6(4), 263-273 (2013)

5. Sang, G.M., Xu, L., de Vrieze, P., Bai, Y., Pan, F.: Predictive maintenance in Industry 4.0. In: ICIST 2020: 10th International Conference on Information Systems and Technologies, 4-5 June 2020

6. Tobon-Mejiaab, D.A., Medjahera, K., Zerhouni, N.: CNC machine tool's wear diagnostic and prognostic by using dynamic Bayesian networks. Mech. Syst. Sig. Process. 28, 167-182 (2012) 
7. Sang, G.M., Xu, L., de Vrieze, P.: Simplifying big data analytics systems with a reference architecture. In: Camarinha-Matos, L.M., Afsarmanesh, H., Fornasiero, R. (eds.) PRO-VE 2017. IAICT, vol. 506, pp. 242-249. Springer, Cham (2017). https://doi.org/10.1007/978-3319-65151-4_23

8. Debevec, M., Simic, M., Herakovic, N.: Virtual factory as an advanced approach for production process optimization. Int. J. Simul. Modell. 13(1), 66-78 (2014)

9. Xu, L., de Vrieze, P., Yu, H., Phalp, K., Bai, Y.: Interoperability of virtual factory: an overview of concepts and research challenges. Int. J. Mech. Manuf. Syst. (2020)

10. Si, X.S., Wang, W., Hu, C.H., Zhou, D.H.: Remaining useful life estimation-a review on the statistical data driven approaches. Eur. J. Oper. Res. 213(1), 1-14 (2011)

11. Lee, J., Baheri, B., Kao, H.: A cyber-physical systems architecture for Industry 4.0-based manufacturing systems. Manuf. Lett. 3, 18-23 (2015)

12. Wang, J., Zhang, L., Duan, L., Gao, R.X.: A new paradigm of cloud-based predictive maintenance for intelligent manufacturing. J. Intell. Manuf. 28(5), 1125-1137 (2017). https://doi.org/10.1007/s10845-015-1066-0

13. Hribernik, K., von Stietencron, M., Bousdekis, A., Bredehorst, B., Mentzas, G., Thoben, K. D.: Towards a unified predictive maintenance system-a use case in production logistics in aeronautics. Procedia Manuf. 16, 131-138 (2018)

14. Guillén, A.J., Crespo, A., Gómez, J.F., Sanz, M.D.: A framework for effective management of condition based maintenance programs in the context of industrial development of EMaintenance strategies. Comput. Ind. 82(2016), 170-185 (2016)

15. FIWARE Catalogue (2020). https://www.fiware.org/developers/catalogue/. Accessed 20 Jan 2020

16. Mathworks (2020). https://www.mathworks.com/help/predmaint/gs/designing-algorithmsfor-condition-monitoring-and-predictive-maintenance.html. Accessed 20 Feb 2020

17. Baruah, P., Chinnam, R.B.: HMMs for diagnostics and prognostics in machining processes. Int. J. Prod. Res. 43(6), 1275-1293 (2005)

18. Bengio, Y., Simard, P., Frasconi, P.: Learning long-term dependencies with gradient descent is difficult. IEEE Trans. Neural Netw. 5(2), 157-166 (1994)

19. Hochreiter, S., Schmidhuber, J.: Long short-term memory. Neural Comput. 9(8), 1735-1780 (1997)

20. Goodfellow, I., Bengio, Y., Courville, A.: Deep Learning. MIT Press, Cambridge (2016)

21. FIWARE Big Data Analysis (2020). https://fiware-tutorials.readthedocs.io/en/latest/big-dataanalysis/index.html. Accessed 20 Feb 2020

22. Apache Hadoop (2020). http://hadoop.apache.org/. Accessed 20 Feb 2020

23. Teti, R., Jemielniak, K., O’Donnell, G., Dornfeld, D.: Advanced monitoring of machining operations. CIRP Ann. 59(2), 717-739 (2010)

24. FIWARE Docker Container Service (2020). https://fiware-docker-container-service.readthed ocs.io/en/latest/index.html. Accessed 20 Feb 2020 